November 1998

SNS-PH/98-24

hep-ph/9811438

IFUP-TH/98-54

\title{
Bubble-Nucleation Rates for Radiatively Induced First-Order Phase Transitions
}

\author{
Alessandro Strumia \\ Dipartimento di Fisica, Università di Pisa and \\ INFN, Sezione di Pisa, I-56127 Pisa, Italia \\ and \\ Nikolaos Tetradis \\ Scuola Normale Superiore, \\ Piazza dei Cavalieri 7, I-56126 Pisa, Italia
}

\begin{abstract}
We present a consistent calculation of bubble-nucleation rates in theories of two scalar fields. Our approach is based on the notion of a coarse-grained free energy that incorporates the effects of fluctuations with momenta above a given scale $k$. We establish the reliability of the method for a variety of two-scalar models and confirm the conclusions of previous studies in one-field theories: Langer's theory of homogeneous nucleation is applicable as long as the expansion around the semiclassical saddle point associated with tunnelling is convergent. This expansion breaks down when the exponential suppression of the rate by the saddle-point action becomes comparable to the pre-exponential factor associated with fluctuations around the saddle point. We reconfirm that Langer's theory is not applicable to the case of weakly first-oder phase transitions. We also find that the same is true in general for radiatively induced first-order phase transitions. We discuss the relevance of our results for the electroweak phase transition and the metastability bound on the Higgs-boson mass.
\end{abstract}




\section{Introduction}

The calculation of bubble-nucleation rates during first-order phase transitions is a difficult problem, both at the conceptual and at the technical level. The standard approach to this problem is based on the work of Langer [1]. His formalism has been applied to relativistic field theory by Coleman [2] and Callan [3] and extended by Affleck [4] and Linde [5] to finite temperature. The nucleation rate $I$ gives the probability per unit time and volume to nucleate a certain region of the stable phase (the true vacuum) within the metastable phase (the false vacuum). Its calculation relies on a semiclassical approximation around a dominant saddle-point, which is identified with the critical bubble. This is a static configuration (usually assumed to be spherically symmetric) within the metastable phase whose interior consists of the stable phase. It has a certain radius that can be determined from the parameters of the underlying theory. Bubbles slightly larger than the critical one expand rapidly, thus converting the metastable phase into the stable one. The nucleation rate is exponentially suppressed by the action (the free energy rescaled by the temperature) of the critical bubble. Possible deformations of the critical bubble generate a pre-exponential factor. The leading contribution to this factor has the form of a fluctuation determinant and corresponds to the first-order correction to the semiclassical result.

For a four-dimensional theory of a real scalar field at temperature $T$, in the limit that thermal fluctuations dominate over quantum fluctuations, the bubble-nucleation rate is given by [3]-[5]

$$
I=\frac{E_{0}}{2 \pi}\left(\frac{S}{2 \pi}\right)^{3 / 2}\left|\frac{\operatorname{det}^{\prime}\left[\delta^{2} \Gamma / \delta \phi^{2}\right]_{\phi=\phi_{\mathrm{b}}}}{\operatorname{det}\left[\delta^{2} \Gamma / \delta \phi^{2}\right]_{\phi=0}}\right|^{-1 / 2} \exp (-S) .
$$

Here $\Gamma$ is the free energy of the system for a given configuration of the field $\phi$ that acts as the order parameter of the problem. The rescaled free energy of the critical bubble is $S=\Gamma_{b} / T=$ $\left[\Gamma\left(\phi_{\mathrm{b}}(r)\right)-\Gamma(0)\right] / T$, where $\phi_{\mathrm{b}}(r)$ is the spherically-symmetric bubble configuration and $\phi=0$ corresponds to the false vacuum. The fluctuation determinants are evaluated either at at $\phi=0$ or around $\phi=\phi_{\mathrm{b}}(r)$. The prime in the fluctuation determinant around the bubble denotes that the three zero eigenvalues of the operator $\left[\delta^{2} \Gamma / \delta \phi^{2}\right]_{\phi=\phi_{\mathrm{b}}}$ have been removed. Their contribution generates the factor $(S / 2 \pi)^{3 / 2}$ and the volume factor that is absorbed in the definition of $I$ (nucleation rate per unit volume). The quantity $E_{0}$ is the square root of the absolute value of the unique negative eigenvalue.

In field theory, the free energy (more precisely the thermodynamic potential) density $\Gamma$ of a system for homogeneous configurations is usually identified with the temperature-dependent effective potential. This is evaluated through some perturbative scheme, such as the loop expansion [6]. The profile and the free energy of the bubble are determined through the potential. This approach, however, faces three fundamental difficulties:

a) The effective potential, being the Legendre transform of the generating functional for the connected Green functions, is a convex function of the field. Consequently, it does not seem to be the appropriate quantity for the study of tunnelling, as no structure with more than one minima separated by a barrier exists. It has been argued in ref. [0] that the appropriate quantity for the study of tunnelling is the generating functional of the 1PI Green functions (calculated perturbatively), which differs from the effective potential in the non-convex regions. However, as we discuss in the following, the consistent picture must rely on the notion of coarse graining and on the separation of the high-frequency fluctuations that may be responsible for the non-convexity of the potential, from the low-frequency ones that are relevant for tunnelling. Such notions cannot be easily implemented in the context of perturbation theory.

b) The fluctuation determinants in the expression for the nucleation rate have a form completely analogous to the one-loop correction to the potential. The question of double-counting the effect of fluctuations (in the potential and the prefactor) must be properly addressed. This point is particularly important in the case of radiatively induced first-order phase transitions. These 
are a consequence of the appearance of a new vacuum state in the theory as a result of the integration of (quantum or thermal) fluctuations [6]. A radiatively induced first-order phase transition takes place in theories for which the tree-level potential has only one minimum, while a second minimum appears at the level of radiative corrections

(c) Another difficulty concerns the ultraviolet divergences that are inherent in the calculation of the fluctuation determinants in the prefactor. An appropriate regularization scheme must be employed in order to control them [9]-[12]. Moreover, this scheme must be consistent with the one employed for the absorption of the divergences appearing in the calculation of the potential that determines the free energy of the critical bubble.

In a previous publication [13], based on the framework described in refs. [14, 15], we demonstrated that all the above issues can be resolved through the implemention of the notion of coarse graining in the formalism. As the appropriate quantity for the description of the physical system we employed the effective average action $\Gamma_{k}$ [16], which is the generalization in the continuum of the blockspin action of Kadanoff [17]. It can be interpreted as a coarse-grained free energy at a given scale $k$. Fluctuations with characteristic momenta $q^{2} \gtrsim k^{2}$ are integrated out and their effect is incorporated in $\Gamma_{k}$. In the limit $k \rightarrow 0, \Gamma_{k}$ becomes equal to the effective action. The $k$ dependence of $\Gamma_{k}$ is described by an exact flow equation [18], typical of the Wilson approach to the renormalization group [19]. This flow equation can be translated into evolution equations for the invariants appearing in a derivative expansion of the action [20, 21]. In ref. [13] we considered only the effective average potential $U_{k}$ and a standard kinetic term and neglected higher derivative terms in the action. We shall employ the same approximation in this paper also. Its validity is guaranteed by the small anomalous dimensions in the models we consider. The bare theory is defined at some high scale $\Lambda$ that can be identified with the ultraviolet cutoff. At scales $k$ below the temperature $T$, the theory can be described in terms of an effective three-dimensional action at zero temperature [22, 23]. This dimensional reduction indicates the absence of explicit time dependence for the parameters of the theory at low energy scales.

In ref. [13] we considered as a starting point the action $\Gamma_{k_{0}}$ for a real scalar field at a scale $k_{0}$ below the temperature, such that the theory has an effective three-dimensional description. We approximated $\Gamma_{k_{0}}$ by a standard kinetic term and a potential with two minima. We assumed that this form of the potential results from the bare potential $U_{\Lambda}$ after the integration of (quantum and thermal) fluctuations between the scales $\Lambda$ and $k_{0}$. Some of these fluctuations may correspond to additional massive degrees of freedom that decoupled above the scale $k_{0}$. We computed the form of the potential $U_{k}$ at scales $k \leq k_{0}$ by integrating an evolution equation derived from the exact flow equation for $\Gamma_{k}$. $U_{k}$ is non-convex for non-zero $k$, and approaches convexity only in the limit $k \rightarrow 0$. The nucleation rate must be computed for $k$ larger than the scale $k_{f}$ at which the functional integral in the definition of $U_{k}$ starts receiving contributions from field configurations that interpolate between the two minima. This happens when $-k^{2}$ becomes approximately equal to the negative curvature at the top of the barrier [24]. For $k>k_{f}$ the typical length scale of a thick-wall critical bubble is $\gtrsim 1 / k$. Through the use of $U_{k}$, the first problem in the calculation of the nucleation rate mentioned above is resolved.

We performed the calculation of the nucleation rate for a range of scales above and near $k_{f}$. In our approach the pre-exponential factor is well-defined and finite, as an ultraviolet cutoff of order $k$ is implemented in the calculation of the fluctuation determinants, such that fluctuations with characteristic momenta $q^{2} \gtrsim k^{2}$ are not included. This is a natural consequence of the fact that all fluctuations

\footnotetext{
${ }^{1}$ In ref. 8] an alternative procedure was suggested for the treatment of radiatively-induced first-order phase transitions: The fields whose fluctuations are responsible for the appearance of the new vacuum are integrated out first, so that an "effective" potential with two minima is generated for the remaining fields. Our philosophy is different: We integrate out high-frequency fluctuations of all fields, so that we obtain an effective low-energy action which we use for the calculation of the nucleation rate. Our procedure involves an explicit infrared cutoff in the calculation of the low-energy action. This prevents the appearance of non-localities arising from integrating out massless fields, which may be problematic for the approach of ref. 8. For example, the fields that generate the new vacuum in radiatively-induced first-order phase transitions are usually massless or very light at the origin of the potential.
} 
with typical momenta above $k$ are already incorporated in the form of $U_{k}$. This modification also resolves naturally the problem of double-counting the effect of the fluctuations.

However, an important issue arises at this point. The scale $k$ was introduced in the problem as a mere calculational tool. If our approach makes sense, the choice of $k$ should not affect physical parameters such as the nucleation rate. The remarkable outcome of our study was that this expectation was confirmed. We found that the saddle-point configuration has an action $S_{k}$ with a significant $k$ dependence. For strongly first-order phase transitions, the nucleation rate $I=A_{k} \exp \left(-S_{k}\right)$ is dominated by the exponential suppression. The main role of the prefactor $A_{k}$, which is also $k$ dependent, is to remove the scale dependence from the total nucleation rate. The implication of our results is that the critical bubble should not be identified just with the saddle point of the semiclassical approximation. It is the combination of the saddle point and its possible deformations in the thermal bath (accounted for by the fluctuation determinant in the prefactor) that has physical meaning. We also found that, for progressively more weakly first-order phase transitions, the difference between $S_{k}$ and $\ln \left(A_{k} / k_{f}^{4}\right)$ diminishes. This indicates that the effects of fluctuations become more and more enhanced. At the same time, a significant $k$ dependence of the predicted nucleation rate develops. The reason for the above deficiency is clear. When the nucleation rate is roughly equal to or smaller than the contribution from the prefactor, the effect of the next order in the expansion around the saddle point is important and can no longer be neglected. This indicates that there is a limit for the validity of Langer's picture of homogeneous nucleation [1].

In this work we describe how our method can be applied to a more complicated system. As such we have chosen a theory of two scalar fields. It provides a framework within which we can test the reliability of our computation of the nucleation rate in the case of two fluctuating fields. The evolution equation for the potential resembles very closely the ones appearing in gauged Higgs theories, with the additional advantage that the approximations needed in the derivation of this equation are more transparent. Moreover, we expect the qualitative conclusions for the region of validity of Langer's picture of homogeneous nucleation to be valid also for gauged Higgs theories. The most interesting feature of the two-scalar models is the presence of radiatively induced first-order phase transitions. Such transitions usually take place when the mass of a certain field is generated through the expectation value of another. The fluctuations of the first field can induce the appearance of new minima in the potential of the second, resulting in first-order phase transitions [6]. As we have already discussed, the problem of double-counting the effect of fluctuations is particularly acute in such situations. The introduction of a coarse-graining scale $k$ resolves this problem, by separating the high-frequency fluctuations of the system which may be responsible for the presence of the second minimum through the Coleman-Weinberg mechanism, from the low-frequency ones which are relevant for tunnelling.

In the following sections we present the calculation of the bubble-nucleation rate for first-order phase transitions in a theory of two real scalar fields. We do not discuss the evolution of $\Gamma_{k}$ for $k \gtrsim T$. We start the evolution at a scale $k_{0}$ sufficiently below the temperature of the system, so that the dynamics is three-dimensional to a good approximation?. As an initial condition we consider a potential $U_{k_{0}}$, whose form is determined by the bare potential $U_{\Lambda}$ and the integration of fluctuations between the scales $\Lambda$ and $k_{0}$. We first establish the reliability of our approach by considering potentials $U_{k_{0}}$ with two minima. Then we turn to the radiatively induced first-order phase transitions, for which the second minimum is generated at some point in the evolution at a scale $k<k_{0}$. In all cases, we integrate the evolution equation for the effective three-dimensional theory starting at the scale $k_{0}$, and perform the calculation of the nucleation rate as described earlier in the introduction.

In the following section we present the model we consider and derive the evolution equation for the potential. In section 3 we summarize the technical points in the calculation of the nucleation rate. Our results are presented in section 4 . The implications of our results for the first-order phase transitions in gauged Higgs models (such as the electroweak phase transition) are given in the conclusions of

\footnotetext{
${ }^{2}$ For readers who are interested in the details of the mechanism of dimensional reduction in our approach, detailed discussions can be found in refs. 22, 23, 26] for a variety of models.
} 
section 5 .

\section{The model and the evolution equation for the potential}

We consider a model of two real scalar fields $\phi_{1}$ and $\phi_{2}$. The effective average action $\Gamma_{k}\left(\phi_{1}, \phi_{2}\right)$ [16] results from the effective integration of degrees of freedom with characteristic momenta larger than a given cutoff $k$. This is achieved by adding an infrared cutoff term to the bare action, so that the effective action does not receive contributions from modes with characteristic momenta $q^{2} \lesssim k^{2}$. In this work we use the simplest choice of a mass-like cutoff term $\sim k^{2}\left(\phi_{1}^{2}+\phi_{2}^{2}\right)$, for which the perturbative inverse propagator for massless fields is $P_{k}(q) \sim q^{2}+k^{2}$. Subsequently, the generating functional for the connected Green functions is defined, from which the generating functional for the 1PI Green functions can be obtained through a Legendre transformation. The presence of the modified propagator in the above definitions results in the effective integration of only the fluctuations with $q^{2} \gtrsim k^{2}$. Finally, the effective average action is obtained by removing the infrared cutoff from the generating functional for the 1PI Green functions.

The effective average action $\Gamma_{k}$ obeys an exact flow equation, which describes its response to variations of the infrared cutoff $k$ [18]. This can be turned into evolution equations for the invariants appearing in a derivative expansion of $\Gamma_{k}$ [20]. In this work we use an approximation which neglects higher derivative terms in the action and approximates it by

$$
\Gamma_{k}=\int d^{3} x\left\{\frac{1}{2}\left(\partial^{\mu} \phi_{1} \partial_{\mu} \phi_{1}+\partial^{\mu} \phi_{2} \partial_{\mu} \phi_{2}\right)+U_{k}\left(\phi_{1}, \phi_{2}\right)\right\} .
$$

The above action describes the effective three-dimensional theory that results from the dimensional reduction of a high-temperature four-dimensional theory at scales below the temperature. The temperature has been absorbed in a redefinition of the fields and their potential, so that these have dimensions appropriate for an effective three-dimensional theory. The correspondence between the quantities we use and the ones of the four-dimensional theory is given by

$$
\phi_{1,2}=\frac{\left[\phi_{1,2}\right]_{4}}{\sqrt{T}}, \quad U\left(\phi_{1}, \phi_{2}\right)=\frac{U_{4}\left(\left[\phi_{1}\right]_{4},\left[\phi_{2}\right]_{4}, T\right)}{T} .
$$

In this way, the temperature does not appear explicitly in our expressions. This has the additional advantage of permitting the straightforward application of our results to the problem of quantum tunnelling in a three-dimensional theory at zero temperature 13.

The evolution equation for the potential can be written in the form [25, 26]

$$
\begin{aligned}
\frac{\partial}{\partial k^{2}}\left[U_{k}\left(\phi_{1}, \phi_{2}\right)-U_{k}(0,0)\right]=-\frac{1}{8 \pi} & {\left[\sqrt{k^{2}+M_{1}^{2}\left(\phi_{1}, \phi_{2}\right)}-\sqrt{k^{2}+M_{1}^{2}(0,0)}+\right.} \\
& \left.+\sqrt{k^{2}+M_{2}^{2}\left(\phi_{1}, \phi_{2}\right)}-\sqrt{k^{2}+M_{2}^{2}(0,0)}\right],
\end{aligned}
$$

where $M_{1,2}^{2}\left(\phi_{1}, \phi_{2}\right)$ are the two eigenvalues of the field-dependent mass matrix, given by

$$
M_{1,2}^{2}\left(\phi_{1}, \phi_{2}\right)=\frac{1}{2}\left[U_{11}+U_{22} \pm \sqrt{\left(U_{11}-U_{22}\right)^{2}+4 U_{12}^{2}}\right]
$$

with $U_{i j} \equiv \partial^{2} U_{k} / \partial \phi_{i} \partial \phi_{j}$. The only neglected corrections to eq. (2.3) are related to the wave-function renormalization of the fields. We expect these correction to be small, as the anomalous dimension is $\eta \approx 0.035-0.04$ for the models we consider and the evolution of the potential takes place over a limited range of $k$. We consider models with the symmetry $\phi_{2} \leftrightarrow-\phi_{2}$ throughout this paper. This means that the expressions for the mass eigenvalues simplify along the $\phi_{1}$-axis: $M_{1}^{2}=\partial^{2} U_{k} / \partial \phi_{1}^{2}$, $M_{2}^{2}=\partial^{2} U_{k} / \partial \phi_{2}^{2}$. We point out that our method does not face the problems of non-convergence of perturbation theory that often appear in the context of radiative symmetry breaking [6]. Up to 
wave-function renormalization effects, eq. (2.3) is exact and its numerical solution is very accurate (see below). However, the inclusion of higher-derivative terms in the action is required for the quantitative study of models with strongly coupled phases, such as the symmetric phase of the electroweak theory above the critical temperature of the electroweak phase transition.

The first step of an iterative solution of eq. (2.3) gives [27]

$$
\begin{aligned}
U_{k}^{(1)}\left(\phi_{1}, \phi_{2}\right)-U_{k}^{(1)}(0)= & \quad U_{k_{0}}\left(\phi_{1}, \phi_{2}\right)-U_{k_{0}}(0)+ \\
+ & \frac{1}{2} \ln \left[\frac{\operatorname{det}\left[-\partial^{2}+k^{2}+M_{1}^{2}\left(\phi_{1}, \phi_{2}\right)\right]}{\operatorname{det}\left[-\partial^{2}+k_{0}^{2}+M_{1}^{2}\left(\phi_{1}, \phi_{2}\right)\right]} \frac{\operatorname{det}\left[-\partial^{2}+k_{0}^{2}+M_{1}^{2}(0)\right]}{\operatorname{det}\left[-\partial^{2}+k^{2}+M_{1}^{2}(0)\right]}\right] \\
+ & \frac{1}{2} \ln \left[\frac{\operatorname{det}\left[-\partial^{2}+k^{2}+M_{2}^{2}\left(\phi_{1}, \phi_{2}\right)\right]}{\operatorname{det}\left[-\partial^{2}+k_{0}^{2}+M_{2}^{2}\left(\phi_{1}, \phi_{2}\right)\right]} \frac{\operatorname{det}\left[-\partial^{2}+k_{0}^{2}+M_{2}^{2}(0)\right]}{\operatorname{det}\left[-\partial^{2}+k^{2}+M_{2}^{2}(0)\right]}\right] .
\end{aligned}
$$

For $k \rightarrow 0$ this is a regularized one-loop approximation to the effective potential. Due to the ratio of determinants, only momentum modes with $k^{2} \lesssim q^{2} \lesssim k_{0}^{2}$ are effectively included in the momentum integrals in eq. (2.5). The above expression demonstrates the form of ultraviolet regularization of fluctuation determinants that is consistent with the cutoff procedure that we described in the beginning of this section in the derivation of the evolution equation for the potential. An analogous regularization will be used in the following section for the fluctuation determinants in the expression for the nucleation rate. The solution of eq. (2.5) also demonstrates that the decoupling of heavy modes is automatically built in the evolution equation for the potential. If $M_{i}^{2}\left(\phi_{1}, \phi_{2}\right) \gg k_{0}^{2} \geq k^{2}$, the contribution of the respective mode to this solution is negligible.

We can determine the symmetries of the theory by specifying the form of the potential at the scale $k_{0}<T$ at which we start the evolution. As we have already mentioned, we assume the symmetry $\phi_{2} \leftrightarrow$ $-\phi_{2}$ throughout this paper. In section 4 we consider an example of a theory without any additional symmetry for the $\phi_{1}$ field. However, for most of our results and especially for those concerning radiatively induced first-order phase transitions, we use potentials invariant under $\phi_{1} \leftrightarrow-\phi_{1}, \phi_{2} \leftrightarrow$ $-\phi_{2}, \phi_{1} \leftrightarrow \phi_{2}$. The solutions of eq. (2.3) preserve the above symmetries for $k<k_{0}$.

For the numerical integration of eq. (2.3), we use the algorithms described in ref. [28], with an appropriate generalization to the two-field case. As we shall be interested in the form of the potential along the $\phi_{1}$-axis, we perform the integration of the evolution equation for $U_{k}\left(\phi_{1}, \phi_{2}\right)$ in a thin region around this axis. In certain cases, which would require excessive computer time, we solve approximate evolution equations for $U_{k}$ and $\partial^{2} U_{k} / \partial \phi_{2}^{2}$ along the $\phi_{1}$-axis $\left(\phi_{2}=0\right)$. We control the accuracy of these approximate solutions by comparing them against the full solution for $U_{k}\left(\phi_{1}, \phi_{2}\right)$ when this is possible. Moreover, we check our numerical results against exact analytical solutions for simplified forms of the evolution equation [29]. We estimate that the numerical uncertainties introduce an error of order $1 \%$ to our results, smaller than the error generated by the omission of the anomalous dimension.

\section{The calculation of the bubble-nucleation rate}

The calculation of the nucleation rate proceeds in complete analogy to the one described in detail in ref. 13] for the one-field case. For this reason, we summarize here only the main steps of the calculation. We consider potentials $U_{k}\left(\phi_{1}, \phi_{2}\right)$ with two minima along the $\phi_{1}$-axis $\left(\phi_{2}=0\right)$ : the stable (true) minimum is located at $\phi_{1}=\phi_{t}$ and the unstable (false) one at $\phi_{1}=\phi_{f}=0$.

The nucleation rate is exponentially suppressed by the action $S_{k}$ (the rescaled free energy) of the saddle-point configuration $\phi_{\mathrm{b}}(r)$ that is associated with tunnelling. This is an $\mathrm{SO}(3)$-invariant solution of the classical equations of motion along the $\phi_{1}$-axis $\left(\phi_{2}=0\right)$ which interpolates between the local maxima of the potential $-U_{k}\left(\phi_{1}\right) \equiv-U_{k}\left(\phi_{1}, \phi_{2}=0\right)$. It satisfies the equation

$$
\frac{d^{2} \phi_{\mathrm{b}}}{d r^{2}}+\frac{2}{r} \frac{d \phi_{\mathrm{b}}}{d r}=\frac{\partial U_{k}\left(\phi_{\mathrm{b}}\right)}{\partial \phi_{1}}
$$


with the boundary conditions $\phi_{\mathrm{b}} \rightarrow 0$ for $r \rightarrow \infty$ and $d \phi_{\mathrm{b}} / d r=0$ for $r=0$. The action $S_{k}$ of the saddle point is given by

$$
S_{k}=4 \pi \int_{0}^{\infty}\left[\frac{1}{2}\left(\frac{d \phi_{\mathrm{b}}(r)}{d r}\right)^{2}+U_{k}\left(\phi_{\mathrm{b}}(r)\right)-U_{k}(0)\right] r^{2} d r .
$$

The profile of the saddle point can be easily computed with the "shooting" method [30]. We integrate eq. (3.1) numerically, starting at $r=0$ with a value of $\phi_{1}$ near the true minimum $\phi_{t}$ and $d \phi_{1} / d r=0$. We then adjust the initial value of $\phi_{1}$ so that the boundary condition $\phi_{\mathrm{b}} \rightarrow 0$ for $r \rightarrow \infty$ is satisfied.

In all the models we consider, the minima of the potential and the saddle-point configuration are located along the $\phi_{1}$-axis $\left(\phi_{2}=0\right)$. The unrenormalized decay rate per unit volume from the false minimum towards the true one is then given by [2, 3, 9 ]

$$
I=\frac{E_{0}}{2 \pi}\left(\frac{S_{k}}{2 \pi}\right)^{3 / 2}\left|\frac{\operatorname{det}^{\prime}\left[\delta^{2} \Gamma_{k} / \delta \phi_{1}^{2}\right]_{\phi_{1}=\phi_{\mathrm{b}}}}{\operatorname{det}\left[\delta^{2} \Gamma_{k} / \delta \phi_{1}^{2}\right]_{\phi_{1}=0}} \frac{\operatorname{det}\left[\delta^{2} \Gamma_{k} / \delta \phi_{2}^{2}\right]_{\phi_{1}=\phi_{\mathrm{b}}}}{\operatorname{det}\left[\delta^{2} \Gamma_{k} / \delta \phi_{2}^{2}\right]_{\phi_{1}=0}}\right|^{-1 / 2} \exp \left(-S_{k}\right) .
$$

This is analogous to eq. (1.1) after the absorption of the explicit factors of $T$ in the redefinition of the fields and potential and the introduction of a coarse-graining scale. If there are several equivalent true vacua, the above rate must be multiplied by an appropriate factor, in order to take into account the possibility of the false vacuum decaying into any of them.

The pre-exponential factor corresponds to the first correction to the semiclassical approximation in the saddle-point method. We are considering models with the symmetry $\phi_{2} \leftrightarrow-\phi_{2}$ throughout this paper. This guarantees that the saddle-point configuration is located along the $\phi_{1}$-axis, where the mass eigenvalues simplify: $M_{1}^{2}=\partial^{2} U_{k} / \partial \phi_{1}^{2} \equiv U_{11}, M_{2}^{2}=\partial^{2} U_{k} / \partial \phi_{2}^{2} \equiv U_{22}$. The numerators in eq. (3.3) are the fluctuation determinants around the saddle-point

$$
\begin{aligned}
\operatorname{det}^{\prime}\left[\delta^{2} \Gamma_{k} / \delta \phi_{1}^{2}\right]_{\phi_{1}=\phi_{\mathrm{b}}} & =\operatorname{det}^{\prime}\left[-\partial^{2}+U_{11}\left(\phi_{1}=\phi_{\mathrm{b}}(r)\right)\right], \\
\operatorname{det}\left[\delta^{2} \Gamma_{k} / \delta \phi_{2}^{2}\right]_{\phi_{1}=\phi_{\mathrm{b}}} & =\operatorname{det}\left[-\partial^{2}+U_{22}\left(\phi_{1}=\phi_{\mathrm{b}}(r)\right)\right],
\end{aligned}
$$

while the denominators are the fluctuation determinants around the false vacuum $\phi_{1}=\phi_{2}=0$

$$
\begin{aligned}
& \operatorname{det}\left[\delta^{2} \Gamma_{k} / \delta \phi_{1}^{2}\right]_{\phi_{1}=0}=\operatorname{det}\left[-\partial^{2}+U_{11}\left(\phi_{1}=0\right)\right], \\
& \operatorname{det}\left[\delta^{2} \Gamma_{k} / \delta \phi_{2}^{2}\right]_{\phi_{1}=0}=\operatorname{det}\left[-\partial^{2}+U_{22}\left(\phi_{1}=0\right)\right] .
\end{aligned}
$$

The differential operator $-\partial^{2}+U_{11}\left(\phi_{\mathrm{b}}(r)\right)$ has three zero modes (the three spatial translations of the critical bubble). The prime over the determinant indicates that these modes have to be omitted in its calculation. Their contribution generates the factor $\left(S_{k} / 2 \pi\right)^{3 / 2}$ in eq. (3.3) and the volume factor that is absorbed in the definition of $I$ (nucleation rate per unit volume). The quantity $E_{0}$ is the square root of the absolute value of the unique negative eigenvalue of the above operator. The operator $-\partial^{2}+U_{22}\left(\phi_{\mathrm{b}}(r)\right)$ has only positive eigenvalues, because the bubble is stable in the $\phi_{2}$ direction at $\phi_{2}=0$.

The pre-exponential factor defined in eq. (3.3) is in general ultraviolet-divergent and an appropriate regularization scheme must be employed. Within our approach, the form of the regularization is dictated by the discussion at the end of the previous section. The effect of the high-frequency modes has been incorporated in the form of the coarse-grained potential $U_{k}$, which is obtained through the integration of the evolution equation (2.3). Fluctuation determinants computed within the low-energy theory must be replaced by a ratio of determinants, in complete analogy to eq. (2.5). This implies that the nucleation rate is given by

$$
I=A_{1 k} A_{2 k} \exp \left(-S_{k}\right)
$$

where 


$$
\begin{aligned}
& A_{1 k}=\frac{E_{0}}{2 \pi}\left(\frac{S_{k}}{2 \pi}\right)^{3 / 2}\left|\frac{\operatorname{det}^{\prime}\left[-\partial^{2}+U_{11}\left(\phi_{\mathrm{b}}(r)\right)\right]}{\operatorname{det}\left[-\partial^{2}+k^{2}+U_{11}\left(\phi_{\mathrm{b}}(r)\right)\right]} \frac{\operatorname{det}\left[-\partial^{2}+k^{2}+U_{11}(0)\right]}{\operatorname{det}\left[-\partial^{2}+U_{11}(0)\right]}\right|^{-1 / 2}, \\
& A_{2 k}=\left|\frac{\operatorname{det}\left[-\partial^{2}+U_{22}\left(\phi_{\mathrm{b}}(r)\right)\right]}{\operatorname{det}\left[-\partial^{2}+k^{2}+U_{22}\left(\phi_{\mathrm{b}}(r)\right)\right]} \frac{\operatorname{det}\left[-\partial^{2}+k^{2}+U_{22}(0)\right]}{\operatorname{det}\left[-\partial^{2}+U_{22}(0)\right]}\right|^{-1 / 2} .
\end{aligned}
$$

The above form of the pre-exponential factors guarantees that only modes with characteristic momenta $q^{2} \lesssim k^{2}$ contribute to the nucleation rate. Another feature of eq. (3.6) is the decoupling of heavy modes with $M_{i}^{2} \gg k^{2}$.

The differential operators that appear in eq. (3.6) have the general form

$$
\mathcal{W}_{i \kappa \alpha}=-\partial^{2}+m_{i \kappa}^{2}+\alpha W_{i k}(r)
$$

where

$$
\begin{aligned}
m_{i \kappa}^{2} & \equiv U_{i i}(0)+\kappa k^{2}, \\
W_{i k}(r) & \equiv U_{i i}\left(\phi_{\mathrm{b}}(r)\right)-U_{i i}(0),
\end{aligned}
$$

with $i=1$ or 2 and $\kappa, \alpha=0$ or 1 . Since the $\mathcal{W}_{i \kappa \alpha}$ operators are $\mathrm{SO}(3)$ symmetric, it is convenient to use spherical coordinates and express the eigenfunctions in terms of spherical harmonics. This leads to

$$
\begin{aligned}
\operatorname{det} \mathcal{W}_{i \kappa \alpha} & =\prod_{\ell=0}^{\infty}\left(\operatorname{det} \mathcal{W}_{i \ell \kappa \alpha}\right)^{2 \ell+1} \\
\mathcal{W}_{i \ell \kappa \alpha} & =-\nabla_{\ell}^{2}+m_{i \kappa}^{2}+\alpha W_{i k}(r),
\end{aligned}
$$

where

$$
\nabla_{\ell}^{2} \equiv \frac{d^{2}}{d r^{2}}-\frac{\ell(\ell+1)}{r^{2}}
$$

and $\ell$ is the usual angular quantum number.

The computation of such complicated determinants is made possible by a theorem [31, 9] that relates ratios of determinants to solutions of ordinary differential equations. In particular, we have

$$
g_{i \ell \kappa} \equiv \frac{\operatorname{det} \mathcal{W}_{i \ell \kappa 1}}{\operatorname{det} \mathcal{W}_{i \ell \kappa 0}}=\frac{\operatorname{det}\left[-\nabla_{\ell}^{2}+m_{i \kappa}^{2}+1 \cdot W_{i k}(r)\right]}{\operatorname{det}\left[-\nabla_{\ell}^{2}+m_{i \kappa}^{2}+0 \cdot W_{i k}(r)\right]}=\frac{y_{i \ell \kappa 1}(r \rightarrow \infty)}{y_{i \ell \kappa 0}(r \rightarrow \infty)}
$$

where $y_{i \ell \kappa \alpha}(r)$ is the solution of the differential equation

$$
\left[-\frac{d^{2}}{d r^{2}}+\frac{\ell(\ell+1)}{r^{2}}+m_{i \kappa}^{2}+\alpha W_{i k}(r)\right] y_{i \ell \kappa \alpha}(r)=0
$$

with the behaviour $y_{i \ell \kappa \alpha}(r) \propto r^{\ell+1}$ for $r \rightarrow 0$. Such equations can be easily solved numerically with Mathematica [32]. The final expression for the nucleation rate, appropriate for an efficient numerical computation 13], is

$$
\begin{aligned}
I & =\frac{1}{2 \pi}\left(\frac{S_{k}}{2 \pi}\right)^{3 / 2} \exp \left(-S_{k}\right) \prod_{i=1}^{2} \prod_{\ell=0}^{\infty} c_{i \ell}, \\
c_{10} & =\left(\frac{E_{0}^{2} g_{101}}{\left|g_{100}\right|}\right)^{1 / 2}, \quad c_{11}=\left(\frac{g_{111}}{g_{110}^{\prime}}\right)^{3 / 2}, \quad c_{1 \ell}=\left(\frac{g_{1 \ell 1}}{g_{1 \ell 0}}\right)^{(2 \ell+1) / 2} \\
c_{2 \ell} & =\left(\frac{g_{2 \ell 1}}{g_{2 \ell 0}}\right)^{(2 \ell+1) / 2}
\end{aligned}
$$



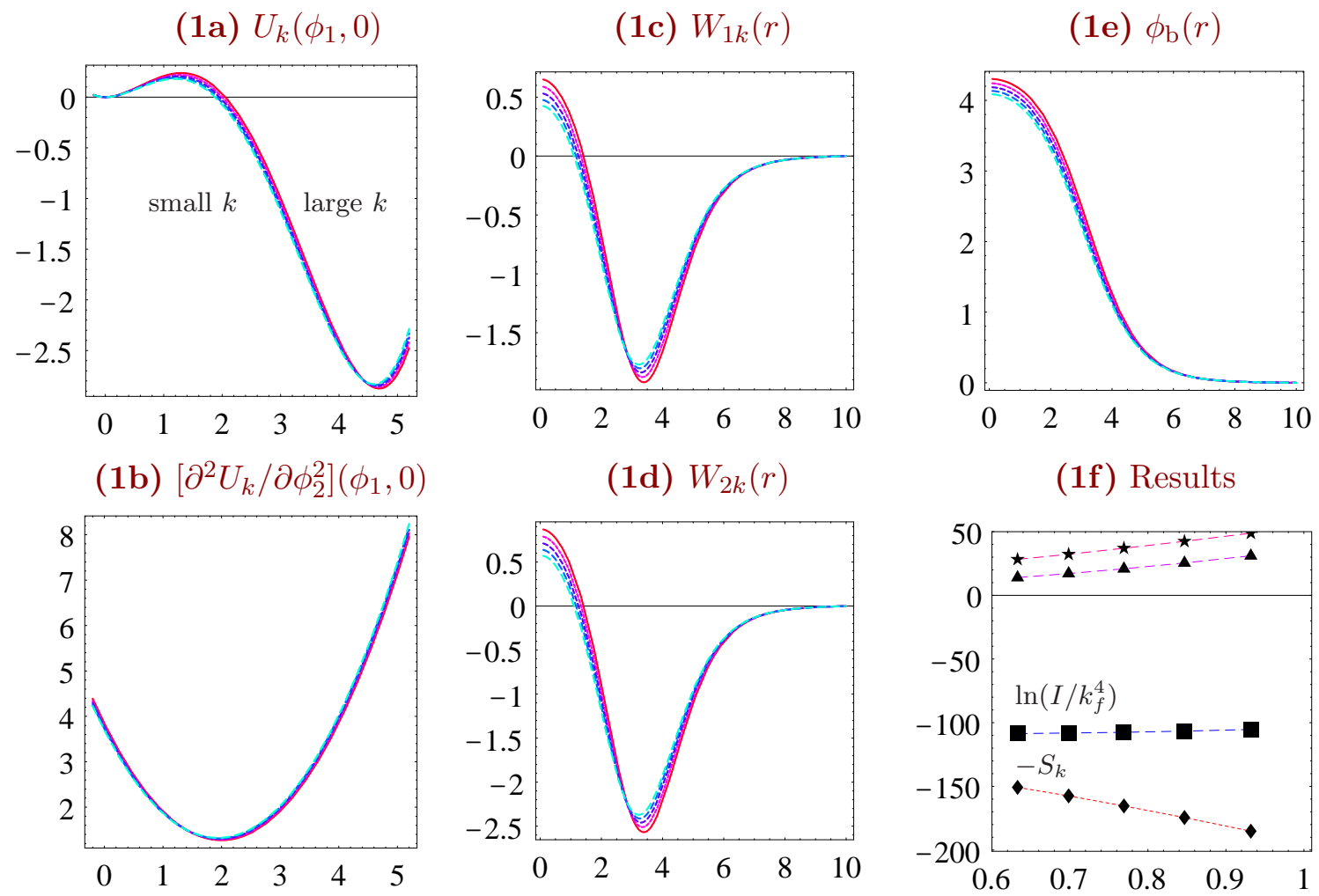

Figure 1: The steps in the computation of the nucleation rate for a model with initial potential given by eq. (4.1) with $m_{2 k_{0}}^{2}=-m_{1 k_{0}}^{2}=0.1 k_{0}^{2}, \lambda_{k_{0}}=g_{k_{0}}=0.1 k_{0}, J_{k_{0}}=0.6 k_{0}^{5 / 2}$. The calculation is performed between the scales $k_{i}=e^{-0.8} k_{0}$ and $k_{f}=e^{-1.2} k_{0}$. All dimensionful quantities are given in units of $k_{f}$. In fig. 1f we plot the saddle-point action (diamonds), the two prefactors $\ln \left(A_{1 k} / k_{f}^{4}\right)$ (stars) and $\ln \left(A_{2 k}\right)$ (triangles), and the nucleation rate $\ln \left(I / k_{f}^{4}\right)$ (squares) as a function of $k / \sqrt{U_{11}\left(\phi_{t}, 0\right)}$.

The calculation of $c_{11}$ is slightly complicated because of the necessity to eliminate the zero eigenvalues in $g_{110}^{\prime}$. Also the (unique) negative eigenvalue $-E_{0}^{2}$ of $\mathcal{W}_{1001}$ must be computed for the determination of $c_{10}$. How these steps are achieved is described in ref. [13], where it is also checked that the product of an infinite number of terms in eq. (3.12) is finite. This can be shown by employing first-order perturbation theory in $W_{i k}$ for large $\ell$, which gives

$$
c_{i \ell} \rightarrow 1+\frac{D_{i}}{\ell^{2}}+\mathcal{O}\left(\ell^{-4}\right), \quad \text { with } \quad D_{i}=-\frac{1}{4} k^{2} \int_{0}^{\infty} r^{3} W_{i k}(r) d r .
$$

As eq. 3.13 gives a reasonable approximation of $c_{i \ell}$ even for small values of $\ell$ in all the cases we consider, we can derive an approximate expression for the prefactors

$$
\ln \left(A_{i k}\right) \approx \operatorname{sign}\left(D_{i}\right) \sqrt{\left|D_{i}\right|} \pi
$$

We have checked that the above expression gives a good analytical approximation to the numerical results we present in the next section.

\section{Results}

As a first example, we apply our formalism to a theory with a potential that strongly resembles the ones used in refs. 13, 25. The potential is consistent with the symmetry $\phi_{2} \leftrightarrow-\phi_{2}$ and near the 

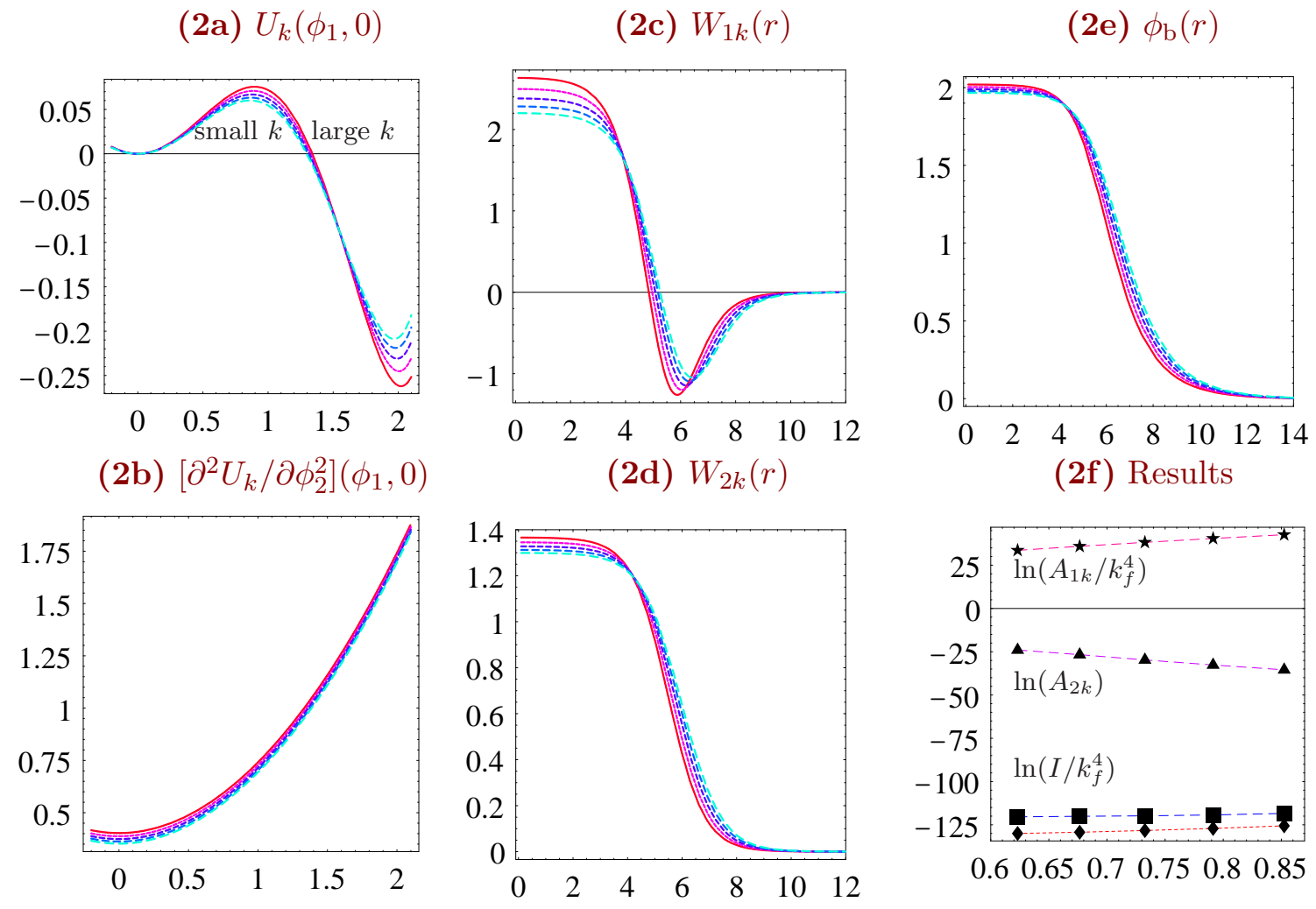

Figure 2: As in fig. 1, but for a model with initial potential given by eq. (4.9) with $\phi_{0 k_{0}}^{2}=2 k_{0}$, $\lambda_{k_{0}}=0.4 k_{0}, g_{k_{0}}=0.3 k_{0}$ and $\nu_{k_{0}}=1$. The calculation is performed between the scales $k_{i}=e^{-0.4} k_{0}$ and $k_{f}=e^{-0.8} k_{0}$. All dimensionful quantities are given in units of $k_{f}$.

$\phi_{1}$-axis has the form

$$
U_{k_{0}}\left(\phi_{1}, \phi_{2}\right)=-J_{k_{0}} \phi_{1}+\frac{1}{2} m_{1 k_{0}}^{2} \phi_{1}^{2}+\frac{1}{2} m_{2 k_{0}}^{2} \phi_{2}^{2}+\frac{1}{8} \lambda_{k_{0}}\left(\phi_{1}^{4}+\phi_{2}^{4}\right)+g_{k_{0}} \phi_{1}^{2} \phi_{2}^{2} .
$$

The term linear in $\phi_{1}$ can be removed through an appropriate shift of $\phi_{1}$ [33, 13]. This would introduce additional terms $\sim \phi_{1}^{3}$ and $\sim \phi_{1} \phi_{2}^{2}$. In fig. 1a we present the evolution of $U_{k}\left(\phi_{1}\right) \equiv U_{k}\left(\phi_{1}, 0\right)$ for $m_{1 k_{0}}^{2}=-0.1 k_{0}^{2}, m_{2 k_{0}}^{2}=0.1 k_{0}^{2}, \lambda_{k_{0}}=g_{k_{0}}=0.1 k_{0}$ and $J_{k_{0}}=0.6 k_{0}^{5 / 2}$. We always shift the location of the false vacuum to zero. The evolution of $U_{22}\left(\phi_{1}\right) \equiv \partial^{2} U_{k} / \partial \phi_{2}^{2}\left(\phi_{1}, 0\right)$ is displayed in fig. $1 \mathrm{~b}$. The solid lines correspond to $k_{i} / k_{0}=e^{-0.8}$, while the line with longest dashes (that has the smallest barrier height) corresponds to $k_{f} / k_{0}=e^{-1.2}$. At the scale $k_{f}$ the negative curvature at the top of the barrier is slightly larger than $-k_{f}^{2}$. This is the point in the evolution of the potential where configurations that interpolate between the minima start becoming relevant in the functional integral that defines the coarse-grained potential [24, 34]. For this reason, we stop the evolution at this point. The potential and the field have been normalized with respect to $k_{f}$, so that they are of order 1 . The profile of the critical bubble $\phi_{\mathrm{b}}(r)$ is plotted in fig. 1e in units of $k_{f}$ for the same sequence of scales. For $k \approx k_{f}$ the characteristic length scale of the bubble profile and $1 / k$ are comparable. This is expected, because the form of the profile is determined by the barrier of the potential, whose curvature is $\approx-k^{2}$ at this point. This is an additional indication that we should not proceed to coarse-graining scales below $k_{f}$. The quantities $W_{1 k}(r)=U_{11}\left(\phi_{\mathrm{b}}(r)\right)-U_{11}(0)$ and $W_{2 k}(r)=U_{22}\left(\phi_{\mathrm{b}}(r)\right)-U_{22}(0)$ are plotted in figs. 1c and $1 \mathrm{~d}$ respectively.

Our results for the nucleation rate are presented in fig. 1f. The horizontal axis corresponds to 

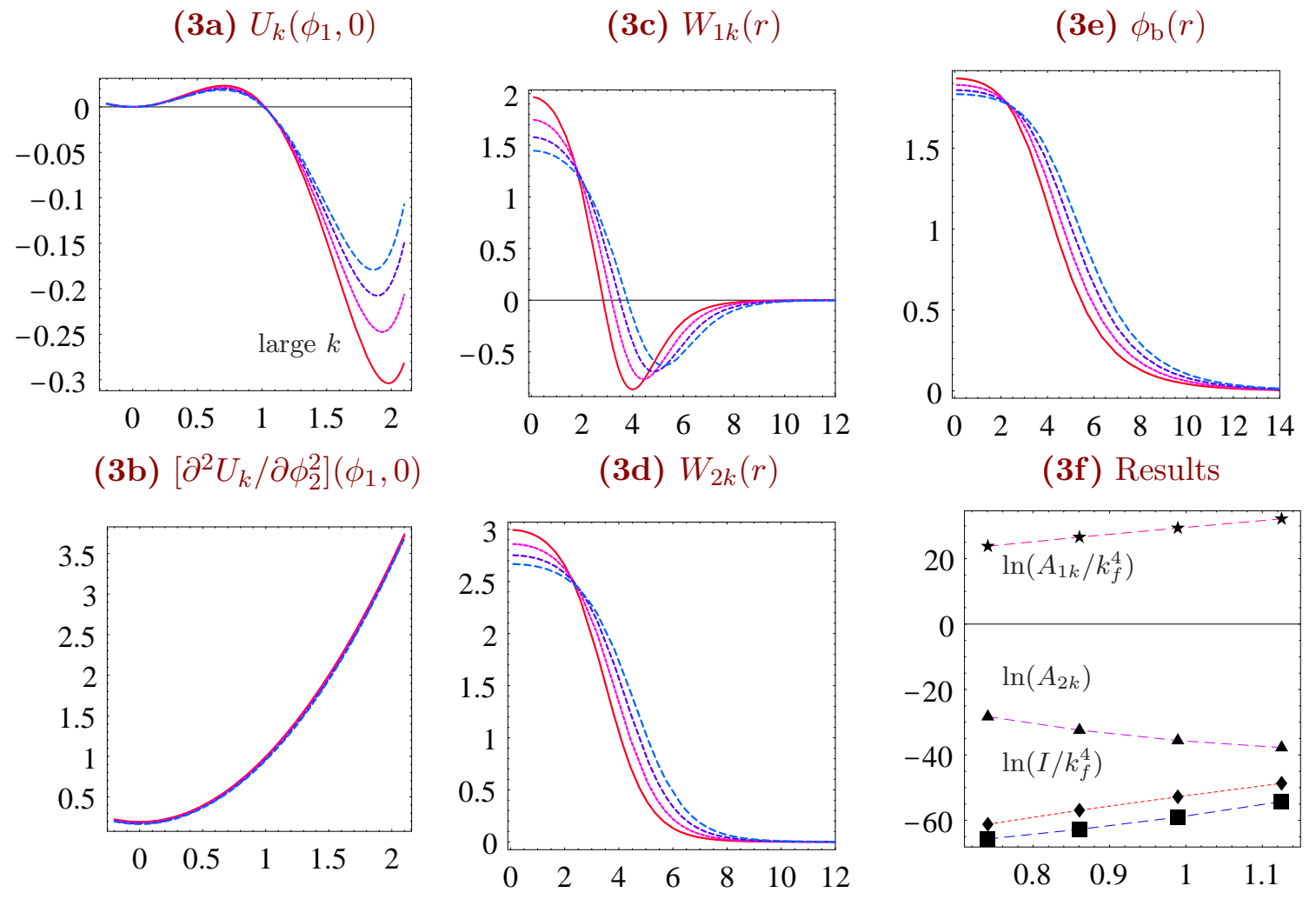

Figure 3: As in fig. 1, but for a model with initial potential given by eq. (4.9) with $\phi_{0 k_{0}}^{2}=2 k_{0}$, $\lambda_{k_{0}}=0.35 k_{0}, g_{k_{0}}=0.8 k_{0}$ and $\nu_{k_{0}}=0.8$. The calculation is performed between the scales $k_{i}=e^{-0.1} k_{0}$ and $k_{f}=e^{-0.7} k_{0}$. All dimensionful quantities are given in units of $k_{f}$.

$\left.k / \sqrt{U_{11}\left(\phi_{t}\right.}\right)$, i.e. the ratio of the scale $k$ to the square root of the positive curvature of the potential along the $\phi_{1}$-axis at the true vacuum. The latter quantity gives the mass of the field $\phi_{1}$ at the absolute minimum. Typically, when $k$ crosses below this mass (corresponding to the value 1 on the horizontal axis) the massive fluctuations of the fields start decoupling (in all the examples we present the mass of $\phi_{2}$ is of the same order or larger than that of $\phi_{1}$ at the absolute minimum) and the evolution of the convex parts of the potential slows down and eventually stops. The dark diamonds give the negative of the action $S_{k}$ of the saddle point at the scale $k$. We observe a strong $k$ dependence of this quantity. The stars in fig. 1d indicate the values of $\ln \left(A_{1 k} / k_{f}^{4}\right)$ and the triangles those of $\ln \left(A_{2 k}\right)$, where the two prefactors $A_{1 k}, A_{2 k}$ are defined in eqs. (3.6). Again a significant $k$ dependence is observed. More specifically, the values of $A_{1 k}$ and $A_{2 k}$ decrease for decreasing $k$. This is expected, because $k$ acts as the effective ultraviolet cutoff in the calculation of the fluctuation determinants. For smaller $k$, fewer fluctuations with wavelengths above an increasing length scale $\sim 1 / k$ contribute explicitly to the fluctuation determinants. We notice that both $A_{1 k}$ and $A_{2 k}$ are significantly smaller than $S_{k}$ and enhance the total nucleation rate. The dark squares give our results for $\ln \left(I / k_{f}^{4}\right)=-S_{k}+\ln \left(A_{1 k} A_{2 k} / k_{f}^{4}\right)$. This quantity has a very small $k$ dependence, which confirms our expectation that the nucleation rate should be independent of the scale $k$. The small residual dependence on $k$ can be used to estimate the contribution of the next order in the expansion around the saddle point. This contribution is expected to be smaller than the first-order correction $\ln \left(A_{1 k} A_{2 k} / k_{f}^{4}\right)$. This example confirms the conclusions of refs. [13, 25] on the region of validity of Langer's theory of homogeneous nucleation: This theory is applicable as long as the expansion around the semiclassical saddle-point is convergent. 


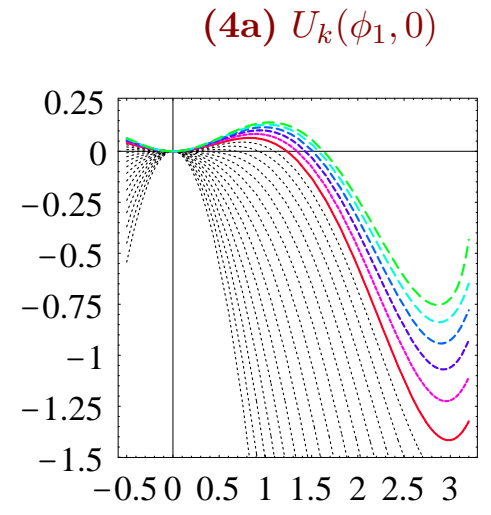

(44اb) $\left[\partial^{2} U_{k} / \partial \phi_{2}^{2}\right]\left(\phi_{1}, 0\right)$

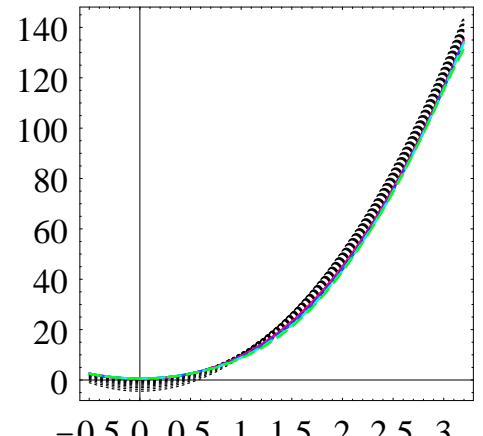

(44) $W_{1 k}(r)$
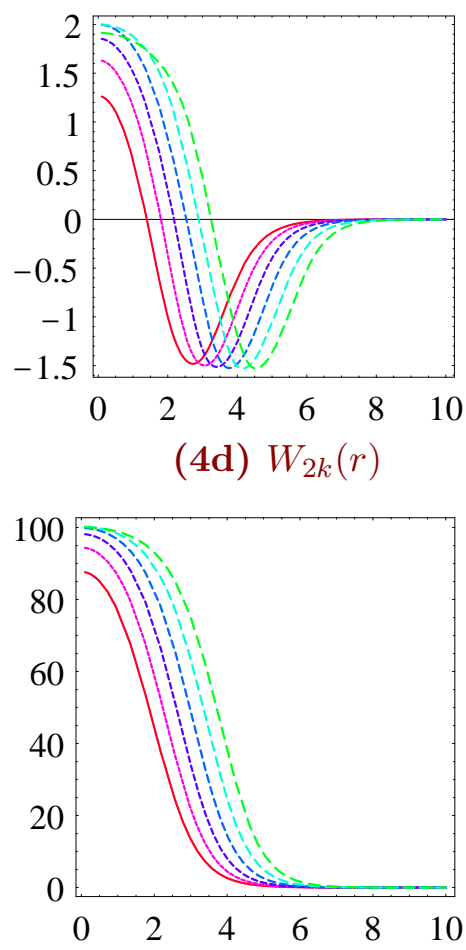

(4e) $\phi_{\mathrm{b}}(r)$
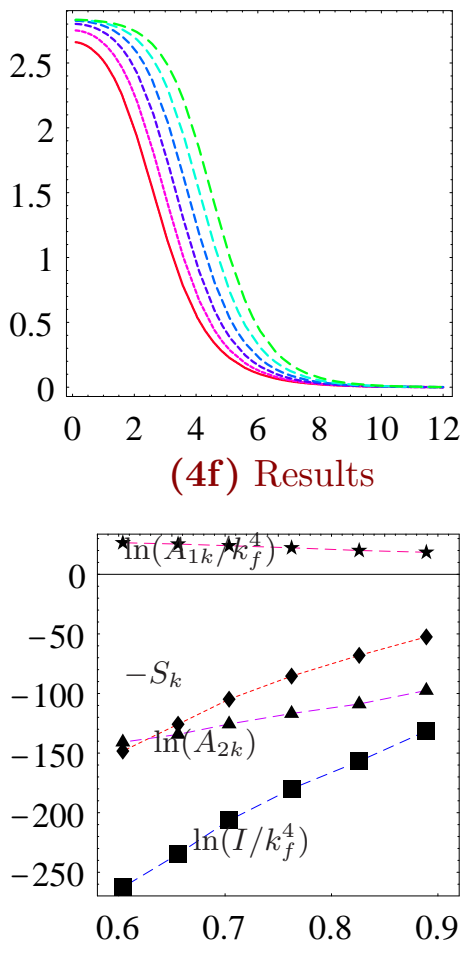

Figure 4: As in fig. 1, but for a radiatively induced first-order phase transition in a model with initial potential given by eq. (4.3) with $\phi_{0 k_{0}}^{2}=1.712 k_{0}, \lambda_{k_{0}}=0.01 k_{0}$ and $g_{k_{0}}=0.2 k_{0}$. The calculation is performed between the scales $k_{i}=e^{-4.7} k_{0}$ and $k_{f}=e^{-5.2} k_{0}$. All dimensionful quantities are given in units of $k_{f}$.

In fig. 2 we consider a theory invariant under the symmetries $\phi_{1} \leftrightarrow-\phi_{1}, \phi_{2} \leftrightarrow-\phi_{2}, \phi_{1} \leftrightarrow \phi_{2}$. The potential is given by

$$
U_{k_{0}}\left(\phi_{1}, \phi_{2}\right)=\frac{\lambda_{k_{0}}}{8}\left[\left(\phi_{1}^{2}-\phi_{0 k_{0}}^{2}\right)^{2}+\left(\phi_{2}^{2}-\phi_{0 k_{0}}^{2}\right)^{2}\right]+\frac{g_{k_{0}}}{4} \phi_{1}^{2} \phi_{2}^{2}+\frac{\nu_{k_{0}}}{48}\left[\left(\phi_{1}^{2}-\phi_{0 k_{0}}^{2}\right)^{3}+\left(\phi_{2}^{2}-\phi_{0 k_{0}}^{2}\right)^{3}\right],
$$

with $\phi_{0 k_{0}}^{2}=2 k_{0}, \lambda_{k_{0}}=0.4 k_{0}, g_{k_{0}}=0.3 k_{0}$ and $\nu_{k_{0}}=1$. In fig. $2 \mathrm{a}$ we present the evolution of $U_{k}\left(\phi_{1}\right)$ and in fig. $2 \mathrm{~b}$ that of $U_{22}\left(\phi_{1}\right)$, in complete analogy to figs. 1a and 1b. The quantities $W_{1 k}(r)=U_{11}\left(\phi_{\mathrm{b}}(r)\right)-U_{11}(0)$ and $W_{2 k}(r)=U_{22}\left(\phi_{\mathrm{b}}(r)\right)-U_{22}(0)$ are plotted in figs. $2 \mathrm{c}$ and $2 \mathrm{~d}$ respectively. Our results for the nucleation rate are shown in fig. 2f. Again, we observe a significant $k$ dependence of $S_{k}, \ln \left(A_{1 k} / k_{f}^{4}\right)$ and $\ln \left(A_{2 k}\right)$ which cancels out in the total rate. A qualitative difference between figs. 1 and 2 concerns the sign of $\ln \left(A_{2 k}\right)$, which is positive in the first case and negative in the second. This behaviour can be understood through the comparison of the form of $W_{2 k}(r)$ in figs. $1 \mathrm{~d}$ and $2 \mathrm{~d}$. In the first case, the mass term of the $\phi_{2}$ fluctuations in most of the interior of the critical bubble is smaller than that in the exterior. As a result, the operator $-\partial^{2}+U_{22}\left(\phi_{\mathrm{b}}(r)\right)$ has lower eigenvalues than the operator $-\partial^{2}+U_{22}(0)$ and the prefactor $A_{2 k}$ in eq. (3.6) tends to enhance the total rate. The opposite is true in the second case. The $\phi_{2}$ fluctuations become more massive in the interior of the bubble and they tend to be suppressed. As these fluctuations are an integral part of the critical bubble, the total rate must be suppressed as well. Notice that the form of $W_{1 k}(r)$ guarantees that the $\phi_{1}$ fluctuations always tend to enhance the nucleation rate.

In fig. 3 we present our results for a potential given by eq. (4.2) with $\phi_{0 k_{0}}^{2}=2 k_{0}, \lambda_{k_{0}}=0.35 k_{0}$, 

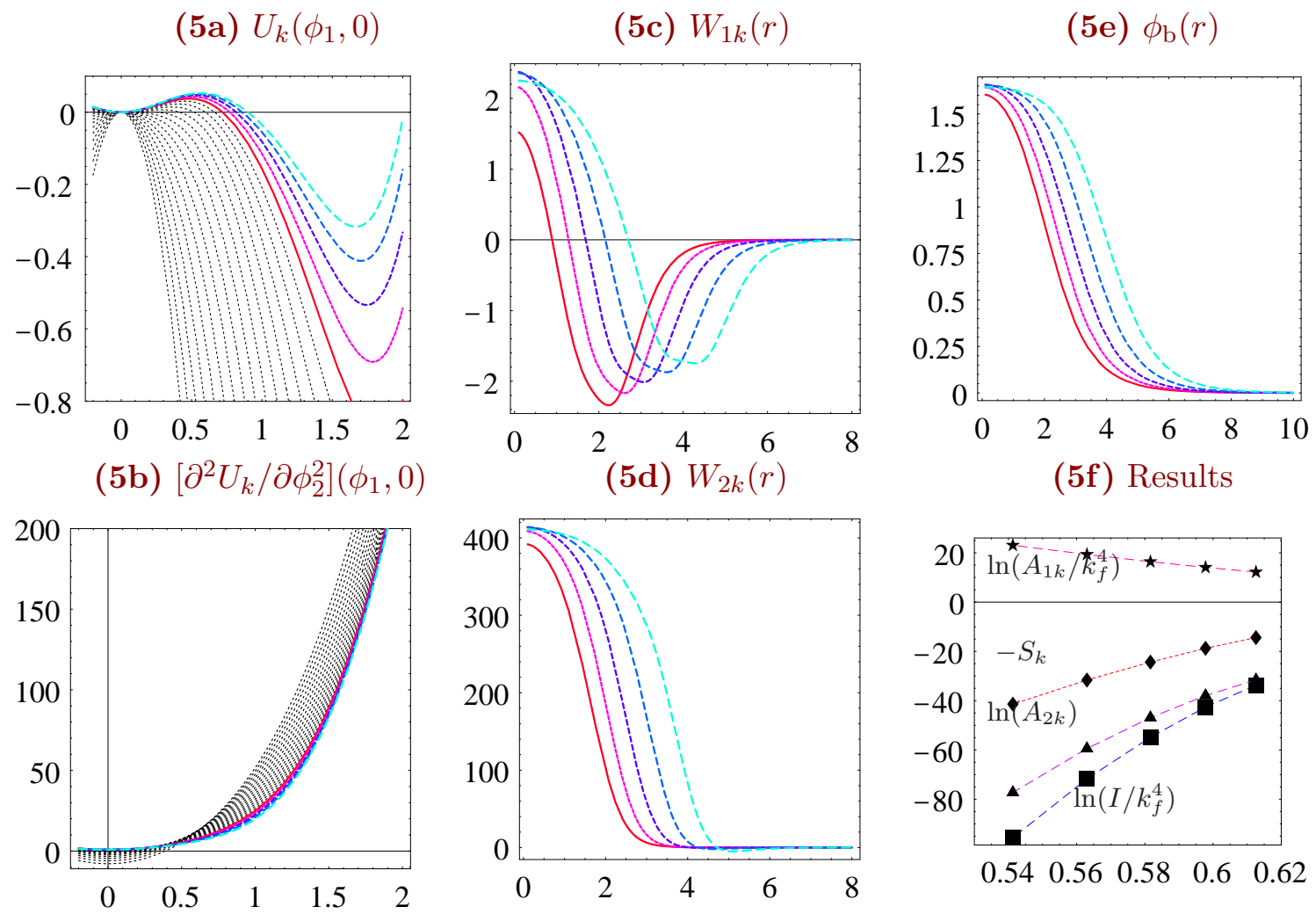

Figure 5: As in fig. 1, but for a radiatively induced first-order phase transition in a model with initial potential given by eq. (4.3) with $\phi_{0 k_{0}}^{2} \approx 0.4999 k_{0}, \lambda_{k_{0}}=0.01 k_{0}$ and $g_{k_{0}}=0.1 k_{0}$. The calculation is performed between the scales $k_{i}=e^{-9.2} k_{0}$ and $k_{f}=e^{-9.6} k_{0}$. All dimensionful quantities are given in units of $k_{f}$.

$g_{k_{0}}=0.8 k_{0}$ and $\nu_{k_{0}}=0.8$. The behaviour is similar to that in fig. 2 , but the first-order corrections $\ln \left(A_{1 k} / k_{f}^{4}\right)$ and $\left|\ln \left(A_{2 k}\right)\right|$ become now comparable to $S_{k}$. This signals the breakdown of the expansion around the semiclassical saddle-point. This is confirmed by the significant $k$ dependence of the predicted total rate, which indicates that the higher-order corrections cannot be neglected anymore. The examples of figs. 1-3 demonstrate the reliability and consistency of our approach in theories with two fluctuating fields. The present work confirms that the picture of tunnelling we have developed is not relevant only for the one-field case studied in refs. 13, 25]. On the contrary, it has a wide range of applicability to many physical systems.

We now turn to the discussion of radiatively induced first-order phase transitions. An example can be observed in a theory defined through the potential

$$
U_{k_{0}}\left(\phi_{1}, \phi_{2}\right)=\frac{\lambda_{k_{0}}}{8}\left[\left(\phi_{1}^{2}-\phi_{0 k_{0}}^{2}\right)^{2}+\left(\phi_{2}^{2}-\phi_{0 k_{0}}^{2}\right)^{2}\right]+\frac{g_{k_{0}}}{4} \phi_{1}^{2} \phi_{2}^{2},
$$

with $\phi_{0 k_{0}}^{2}=1.712 k_{0}, \lambda_{k_{0}}=0.01 k_{0}$ and $g_{k_{0}}=0.2 k_{0}$. Our results for this theory are presented in fig. 4. In fig. 4 a we plot a large part of the evolution of $U_{k}\left(\phi_{1}\right)$. The initial potential has only one minimum along the positive $\phi_{1}$-axis (and the equivalent ones under the the symmetries $\phi_{1} \leftrightarrow-\phi_{1}$, $\phi_{2} \leftrightarrow-\phi_{2}, \phi_{1} \leftrightarrow \phi_{2}$ ) and a maximum at the origin. In the sequence of potentials depicted by dotted lines we observe the appearance of a new minimum at the origin at some point in the evolution (at $k / k_{0} \approx e^{-4.4}$ ). This minimum is generated by the integration of fluctuations of the $\phi_{2}$ field, whose mass depends on $\phi_{1}$ through the last term in eq. (4.3) (the Coleman-Weinberg mechanism). 
Detailed descriptions of this phenomenon within the framework of the effective average action have been presented in refs. [14, 15, 23, 26, 35, 36] for a variety of models. In fig. 4b it can be seen that the mass term of the $\phi_{2}$ field at the origin turns positive at the same value of $k$. This is a consequence of the $\phi_{1} \leftrightarrow \phi_{2}$ symmetry of the potential. We calculate the nucleation rate using the potentials of the last stages of the evolution. The solid lines correspond to $k_{i} / k_{0}=e^{-4.7}$, while the line with longest dashes corresponds to $k_{f} / k_{0}=e^{-5.2}$. In figs. $4 \mathrm{~b}-4 \mathrm{e}$ we observe that the mass of the $\phi_{2}$ fluctuations in the interior of the critical bubble is much larger than the other mass scales of the problem, which are comparable to $k_{f}$. This is a consequence of our choice of couplings $g / \lambda=20$. Such a large ratio of $g / \lambda$ is necessary for a strongly first-order phase transition to be radiatively induced. Unfortunately, this range of couplings also leads to large values for the $\phi_{2}$ mass and, as a result, to values of $\left|\ln \left(A_{2 k}\right)\right|$ that are comparable or larger than the saddle-point action $S_{k}$, even though $\ln \left(A_{1 k} / k_{f}^{4}\right)$ remains small. As a result, the saddle-point approximation breaks down and the predicted nucleation rate $I / k_{f}^{4}$ is strongly $k$-dependent.

It may be possible to obtain a convergent expansion around the saddle point by considering models with smaller values of $g$. In fig. 5 we repeat the calculation of the nucleation rate for a theory described by the potential of eq. (4.3) with $\phi_{0 k_{0}}^{2} \approx 0.4999 k_{0}, \lambda_{k_{0}}=0.01 k_{0}$ and $g_{k_{0}}=0.1 k_{0}$. Due to the smaller value of the ratio $g / \lambda=10$, we observe a more weakly first-order phase transition. This can be checked by considering the value of $k_{f}$ that sets the scale for the dimensionful quantities in figs. 4 and 5. This scale is smaller by a factor $e^{-4.4} \approx 0.012$ in fig. 5 than in fig. 4 . Fig. $5 \mathrm{f}$ indicates that the expansion around the saddle point is more problematic in this case. Not only $\left|\ln \left(A_{2 k}\right)\right|$ is larger than the saddle-point action $S_{k}$, but the prefactor $\ln \left(A_{1 k} / k_{f}^{4}\right)$, associated with the fluctuations of the $\phi_{1}$ field, becomes now comparable to $S_{k}$. This behaviour of $A_{1 k}$ for weakly first-order phase transitions has already been observed in refs. [13, 25]. We have not managed to find any region of the parameter space that leads to a convergent saddle-point expansion for the nucleation rate. The generic behaviour resembles either the one depicted in fig. 4 for strongly first-order phase transitions, or the one in fig. 5 for weakly first-order phase transitions. The implications for cosmological phase transitions, such as the electroweak, are discussed in the following section.

\section{Discussion and conclusions}

In this paper we generalized to theories of two scalar fields the analysis of refs. [13, 25] for the consistent description of first-order phase transitions in the one-field case. We established the reliability of the method by making consistency checks for a variety of models described by different potentials. The conclusions of refs. 13, 25] were verified in this more general context: Langer's theory of homogeneous nucleation is applicable as long as the expansion around the semiclassical saddle point that dominates tunnelling is convergent. The total bubble-nucleation rate is not determined simply through the exponential suppression by the action of the saddle point. The fluctuations around the saddle point are important and generate a pre-exponential factor which can be significant even in the cases for which the saddle-point expansion is convergent. In the example of fig. 1 the logarithm of the pre-exponential factor is about $30 \%$ of the saddle-point action.

If the prefactor becomes comparable to the saddle-point action, the expansion ceases to converge. In our approach this is signalled by the appearance of a strong dependence of the predicted nucleation rate on the coarse-graining scale at which the free energy of the system is evaluated. We found two types of situations in which this happens:

a) The fluctuations of the field $\phi_{1}$, that serves as the order parameter for the phase transition and varies along the saddle point, become significant in the case of weakly first-order phase transitions. Typically these fluctuations enhance the total rate and can even compensate the exponential suppression. Several examples of this behaviour were given in refs. [13, 25]. The conclusions were reconfirmed by the study of the prefactor $A_{1 k}$ in fig. 5. The reason for this behaviour can be traced to the form of the differential operators in the prefactor associated with 
the field $\phi_{1}$ (see eqs. eqs. (3.6) $\left.-(\sqrt{3.7 \mathrm{c}})\right)$. This prefactor, before regularization, involves the ratio $\operatorname{det}^{\prime}\left(-\partial^{2}+m_{1}^{2}+W_{1 k}(r)\right) / \operatorname{det}\left(-\partial^{2}+m_{1}^{2}\right)$, with $m_{1}^{2}=U_{11}(0)$ and $W_{1 k}(r)=U_{11}\left(\phi_{b}(r)\right)-U_{11}(0)$. The function $W_{1 k}(r)$ always has a minimum away from $r=0$ (see figs. 1-5), where it takes negative values. As a result the lowest eigenvalues of the operator $\operatorname{det}^{\prime}\left(-\partial^{2}+m_{1}^{2}+W_{1 k}(r)\right)$ are smaller than those of $\operatorname{det}\left(-\partial^{2}+m_{1}^{2}\right)$. The elimination of the very large eigenvalues from the determinants through regularization does not affect this fact and the prefactor $A_{1 k}$ is always larger than 1. Moreover, for weakly first-order phase transitions it becomes exponentially large because of the proliferation of low eigenvalues in $\operatorname{det}^{\prime}\left(-\partial^{2}+m_{1}^{2}+W_{1 k}(r)\right)$. In physical terms, this implies the existence of a large class of field configurations of free energy comparable to that of the saddle-point. Despite the fact that they are not saddle points of the free energy (they are rather deformations of a saddle point) and are, therefore, unstable, they result in a dramatic increase of the nucleation rate. This picture is very similar to that of "subcritical bubbles" of ref. [37]. In ref. [38] the nucleation rate was computed by first calculating a corrected potential that incorporates the effect of such non-perturbative configurations. The pre-exponential factor must be assumed to be of order 1 in this approach, as the effect of most deformations of the critical bubble has already been taken into account in the potential. In our approach the non-perturbative effects are incorporated through the prefactor. Both methods lead to similar conclusions for the enhancement of the total nucleation rate.

b) The fluctuations of the field $\phi_{2}$, that is orthogonal to $\phi_{1}$ and can trigger a radiatively induced first-order phase transition, can also become important. In the radiatively induced scenario, the mass of $\phi_{2}$ depends on the expectation value of $\phi_{1}$ through a mixing term $\sim g \phi_{1}^{2} \phi_{2}^{2}$ in the potential. If the coupling $g$ is sufficiently large, a second minimum of the renormalized potential can be generated through the integration of the $\phi_{2}$ fluctuations, even if the bare potential has only one minimum. In all cases where this was achieved in the models we investigated (figs. 4 and 5 and results not presented here), we found that the pre-exponential factor is large compared to the saddle-point action. This is not surprising. The radiative corrections to the potential and the pre-exponential factor have a very similar form of fluctuation determinants. When the radiative corrections are large enough to modify the bare potential and generate a new minimum, the pre-exponential factor should be expected to be important also. More precisely, the reason for this behaviour can be traced again to the form of the differential operators in the prefactor. The prefactor associated with the field $\phi_{2}$ involves the ratio $\operatorname{det}\left(-\partial^{2}+m_{2}^{2}+W_{2 k}(r)\right) / \operatorname{det}\left(-\partial^{2}+m_{2}^{2}\right)$, with $m_{2}^{2}=U_{22}(0)$ and $W_{2 k}(r)=U_{22}\left(\phi_{b}(r)\right)-U_{22}(0)$. In units in which $\phi_{b}(r)$ is of order 1 , the function $W_{2 k}(r)$ takes very large positive values near $r=0$ (see figs. 4,5). This is a consequence of the large values of $g$ that are required for the appearance of a new minimum in the potential. As a result, the lowest eigenvalues of the operator $\operatorname{det}\left(-\partial^{2}+m_{2}^{2}+W_{2 k}(r)\right)$ are much larger than those of $\operatorname{det}\left(-\partial^{2}+m_{2}^{2}\right)$. This induces a large suppression of the nucleation rate. In physical terms, this implies that the deformations of the critical bubble in the $\phi_{2}$ direction cost excessive amounts of free energy. As these fluctuations are inherent to the system, the total nucleation rate is suppressed when they are taken into account properly.

An interesting question concerns the possibility to ameliorate the situation for the radiatively induced first-order phase transitions by integrating out completely the $\phi_{2}$ field along the lines of ref. [8]. One may even envisage theories in which the term $\sim \phi_{2}^{4}$ is absent and the gaussian integration over $\phi_{2}$ can be peformed exactly. The result would be the appearance of a term $\sim \ln \operatorname{det}\left(-\partial^{2}+U_{22}\left(\phi_{1}\right)\right)$ in the "effective" action of the $\phi_{1}$ field. For homogeneous field configurations, such a term would generate the two-minimum structure in the effective potential. However, the determination of the bubble profile would require the minimization of the whole "effective" action, including the additional term $\sim \ln \operatorname{det}\left(-\partial^{2}+U_{22}\left(\phi_{1}\right)\right)$. The evaluation of the prefactor would involve a contribution that corresponds to a higher order in the semiclassical expansion that we employed in this work. The reason is that ln $\operatorname{det}\left(-\partial^{2}+U_{22}\left(\phi_{1}\right)\right)$ must be expanded around the saddle point configuration $\phi_{b}(r)$ and the new $\phi_{1}$ integration must be performed. Thus, it is clear that this approach does not offer any technical 
advantages. Moreover, it is possible that an action that includes the term $\sim \ln \operatorname{det}\left(-\partial^{2}+U_{22}\left(\phi_{1}\right)\right)$ is problematic due to the appearance of non-localities for field configurations for which $U_{22}\left(\phi_{1}\right) \approx 0$.

Our approach is based on a separation of the problem in two steps. At first, the high frequency modes of all fields are integrated out. The resulting effective theory can be approximated by a local action, as the integration of modes is performed with an explicit infrared cutoff so that non-localities are suppressed. Subsequently, the nucleation problem is addressed in a systematic expansion around the saddle point. A possibility suggested by the discussion in the previous paragraph is that an "improved" saddle point may be obtained if we minimize $S_{k}+\ln A_{k}$ instead of only $S_{k}$. However, the corrections to the total rate that are induced by this change are of the same order as corrections from going beyond the gaussian approximation in the expansion around the saddle point. This has been confirmed by approximating $\ln A_{k}$ through eq. (3.14) and minimizing $S_{k}+\ln A_{k}$. For $\left|\ln A_{k}\right|$ smaller than $S_{k}$ we have found that, although the saddle point is modified, the value of $S_{k}+\ln A_{k}$ remains largely unaffected.

Our results are relevant for most cosmological phase transitions. For example, the electroweak phase transition for Higgs boson masses below $\approx 80 \mathrm{GeV}$ is a radiatively induced first-order one. The same is true for the phase transitions in extensions of the standard model or in grand unified theories. Usually the second minimum of the potential of the scalar Higgs field appears as a result of the integration of the fluctuations of gauge fields, whose mass is generated through the Higgs mechanism. There is a close resemblance with the two-scalar models we considered. This is apparent if one considers the mass term of the $\phi_{2}$ field along the $\phi_{1}$-axis. For the potential of eq. (4.3), it is initially given by $M_{2 k_{0}}^{2}\left(\phi_{1}\right)=-\lambda_{k_{0}} \phi_{0 k_{0}}^{2} / 2+g_{k_{0}} \phi_{1}^{2} / 2$. For the range of couplings $g_{k_{0}} \gg \lambda_{k_{0}}$, which is relevant for radiatively induced first-order phase transitions, the first term in the previous expression is negligible. The evolution of the potential at scales $k<k_{0}$ results in the replacement of $g_{k_{0}}$ by an effective running coupling $g_{k}\left(\phi_{1}\right)$. However, in cases similar to the ones we considered, in which no fixed points affect the evolution, the running of $g$ is not substantial.

In the gauged Higgs models, the mass of the gauge fields is generated through a term $\sim e^{2} \phi_{1}^{2} A^{\mu} A_{\mu}$. At the level of the bare action the mass term is $M_{g k_{0}}^{2}\left(\phi_{1}\right)=e_{k_{0}}^{2} \phi_{1}^{2}$. Within a truncation that preserves the same invariants as the bare action, the evolution of the potential is given by an equation completely analogous to eq. (2.3) with $M_{2}^{2}$ replaced by $M_{g}^{2}$ (and the modification of some numerical factors) [39, 23, 36, 40]. The replacement of $e_{k_{0}}^{2}$ by a running coupling $e_{k}^{2}\left(\phi_{1}\right)$ at scales $k<k_{0}$ does not modify the qualitative behaviour of the potential, as long as a first-order phase transition takes place [23. It is very likely, therefore, that our conlcusions concerning the convergence of the expansion around the saddle point remain valid in the case of the radiatively induced first-order phase transitions in the gauged Higgs models. This would imply that the estimates of the nucleation rate that rely only on the exponential suppression factor may be very misleading. Moreover, the whole series may not be convergent and this would make an alternative calculational scheme necessary.

A confirmation of the above possibility is provided by estimates of the bubble-nucleation rate based on a perturbative approximation of the effective action. Such a calculation was performed in ref. [11] for the electroweak phase transition. An effective action for the Higgs field was obtained by integrating out the gauge fields through a perturbative calculation. The saddle-point action was evaluated within the effective scalar theory in the standard way, while the pre-exponential factor was computed using the heat-kernel method. There are several points that need refinement in this approach: a) It is questionable whether the gauge fields can be integrated out perturbatively, as they are massless in the symmetric phase within perturbation theory. b) The derivative expansion of the effective action for the scalar fields is problematic around the origin of the potential, where the gauge theory becomes strongly coupled. c) In the absence of a coarse-graining scale the fluctuations of the scalar fields cannot be treated properly, as the problem of double-counting their effect in the effective action and the prefactor is not resolved. It is remarkable, however, that, despite the above problems, the conclusions of ref. [11] are in agreement with the implications of our results: The pre-exponential factor is significant and results in the additional suppression of the total rate. Moreover, the saddle-point approximation is problematic near the critical temperature. 
The application of our formalism to the electroweak phase transition must deal with several technical points. Firstly, an efficient truncation of the average effective action must be developed, appropriate for the discussion of gauged Higgs theories. Such truncations have been discussed in refs. [39, 23, 36, 40. and the resulting evolution equations for the potential and the gauge coupling have been obtained. In the case of the $S U(2)$ Higgs model, they account for the growth of the gauge coupling near the origin of the potential and the emergence of a strongly coupled regime. They also take into account the contribution of the Higgs-field fluctuations. The saddle-point action can be evaluated along the lines of refs. 14, 15, 13 and the present work. The calculation of the pre-exponential factor is more difficult. The fluctuation determinants associated with the gauge fields must be computed in complete analogy to those of the $\phi_{2}$ field in this work. This means that they must be evaluated in the background of the saddle point, with an ultraviolet regularization that matches the cutoff procedure in the derivation of the evolution equations for the potential and the gauge couplings. Particular care is required for the treatment of additional zero eigenvalues that result from the presence of Goldstone modes in the phase with symmetry breaking [41]. However, we believe that the above technical points should not affect the qualitative conclusion reached in this work: If the radiative corrections can affect the vacuum structure of the theory, the pre-exponential factor in the tunnelling rate is significant.

Another possible application concerns the bound on the Higgs-boson mass from the requirement that our vacuum is not destabilized by the presence of a non-standard vacuum at large Higgs-field values [42, 43. This new vacuum is generated by the top-quark radiative corrections to the effective potential. The most stringent bound arises from the requirement that the probability of thermal tunnelling from the symmetric phase to the non-standard vacuum at some temperature above the critical temperature of the electroweak phase transition is negligible. This problem can be treated within an effective three-dimensional theory at energy scales below the temperature. At such scales the top quark can be integrated out, as its propagator contains an effective mass of the order of the temperature. Its effect can be incorporated in the initial conditions for the evolution equations for the potential and the gauge couplings. The potentials that are relevant for this problem have a form very close to that along the spinodal line (the false vacuum is at the end of the metastability range and very close to becoming unstable) 443]. It is, therefore, conceivable that the prefactor generated by the Higgs-field fluctuations is significant [25]. The effect of the gauge-field fluctuations must be taken into account as well, in analogy to the calculation of the nucleation rate for the electroweak phase transition.

Acknowledgements We would like to thank R. Barbieri, J. Espinosa and C. Wetterich for helpful discussions. The work of N.T. was supported by the E.C. under TMR contract No. ERBFMRXCT96-0090.

\section{References}

[1] J. Langer, Ann. Phys. 41, 108 (1967); ibid. 54, 258 (1969); Physica 73, 61 (1974).

[2] S. Coleman, Phys. Rev. D 15, 2929 (1977).

[3] C.G. Callan and S. Coleman, Phys. Rev. D 16, 1762 (1977).

[4] I. Affleck, Phys. Rev. Lett. 46, 388 (1981).

[5] A.D. Linde, Nucl. Phys. B 216, 421 (1983).

[6] S. Coleman and E. Weinberg, Phys. Rev. D 7, 1888 (1973).

[7] E. Weinberg and A. Wu, Phys. Rev. D 36, 2474 (1987).

[8] E. Weinberg, Phys. Rev. D 47, 4614 (1993). 
[9] W.N. Cottingham, D. Kalafatis and R. Vinh Mau, Phys. Rev. B 48, 6788 (1993).

[10] J. Baacke and V.G. Kiselev, Phys. Rev. D 48, 5648 (1993); J. Baacke, ibid. 52, 6760 (1995).

[11] J. Kripfganz, A. Laser and M.G. Schmidt, Nucl. Phys. B 433, 467 (1995).

[12] M. Gleiser, G.C. Marques and R.O. Ramos, Phys. Rev. D 48, 1571 (1993); G.H. Flores, R.O. Ramos and N.F. Svaiter, preprint hep-th/9903009.

[13] A. Strumia and N. Tetradis, preprint SNS-PH/98-12, hep-ph/9806453.

[14] J. Berges, N. Tetradis and C. Wetterich, Phys. Lett. B 393, 387 (1997).

[15] J. Berges and C. Wetterich, Nucl. Phys. B 487, 675 (1997).

[16] C. Wetterich, Nucl. Phys. B 352, 529 (1991); Z. Phys. C 57, 451 (1993); it ibid. 60, 461 (1993).

[17] L.P. Kadanoff, Physics 2, 263 (1966).

[18] C. Wetterich, Phys. Lett. B 301, 90 (1993).

[19] K.G. Wilson, Phys. Rev. B 4, 3174 and 3184 (1971); K.G. Wilson and I.G. Kogut, Phys. Rep. 12, 75 (1974); F.J. Wegner, in: Phase Transitions and Critical Phenomena, vol. 6, eds. C. Domb and M.S. Green (Academic Press, New York, 1976).

[20] N. Tetradis and C. Wetterich, Nucl. Phys. B 422, 541 (1994).

[21] T.R. Morris, Phys. Lett. B 329, 241 (1994).

[22] N. Tetradis and C. Wetterich, Nucl. Phys. B 398, 659 (1993); Int. J. Mod. Phys. A 9, 4029 (1994).

[23] N. Tetradis, Nucl. Phys. B 488, 92 (1997).

[24] A. Ringwald and C. Wetterich, Nucl. Phys. B 334, 506 (1990); N. Tetradis and C. Wetterich, Nucl. Phys. B 383, 197 (1992).

[25] A. Strumia, N. Tetradis and C. Wetterich, preprint SNS-PH/98-13, hep-ph/9808263.

[26] S. Bornholdt, N. Tetradis and C. Wetterich, Phys. Lett. B 348, 89 (1995); Phys. Rev. D 53, 4552 (1996); S. Bornholdt, P. Büttner, N. Tetradis and C. Wetterich, preprint CERN-TH/96-67, cond-mat/9603129; N. Tetradis, Phys. Lett. B 431, 380 (1998).

[27] C. Wetterich, Mod. Phys. Lett. A 11, 2573 (1996).

[28] J. Adams, J. Berges, S. Bornholdt, F. Freire, N. Tetradis and C. Wetterich, Mod. Phys. Lett. A 10, 2367 (1995).

[29] N. Tetradis and D. Litim, Nucl. Phys. B 464, 492 (1996).

[30] W.H. Press, B.P. Flannery, S.A. Teukolsky and W.T. Vetterling, Numerical Recipes: The Art of Scientific Computing (University Press, Cambridge, 1988).

[31] S. Coleman, in The Whys of Subnuclear Physics, Proceedings of the International School, Erice, Italy, 1977, ed. by A. Zichichi, Subnuclear Series Vol. 15 (Plenum, New York, 1979).

[32] S. Wolfram, The Mathematica book, 3rd ed. (Wolfram Media/Cambridge University Press, 1996).

[33] S. Seide and C. Wetterich, preprint HD-THEP-98-20, cond-mat/9806372. 
[34] J. Alexandre, V. Branchina and J. Polonyi, preprint cond-mat/9803007.

[35] D. Litim, C. Wetterich and N. Tetradis, Mod. Phys. Lett. A 12, 2287 (1997).

[36] B. Bergerhoff, D. Litim, S. Lola and C. Wetterich, Int. J. Mod. Phys. A 11, 4273 (1996); B. Bergerhoff, F. Freire, D. Litim, S. Lola and C. Wetterich, Phys. Rev. B 53, 5734 (1996).

[37] M. Gleiser, E.W. Kolb and R. Watkins, Nucl. Phys. B 364, 411 (1991); M. Gleiser and E.W. Kolb, Phys. Rev. Lett. 69, 1304 (1992); Phys. Rev. D 48, 1560 (1993); N. Tetradis, Z. Phys. C 57, 331 (1993); G. Gelmini and M. Gleiser, Nucl. Phys. B 419, 129 (1994); M. Gleiser, Phys. Rev. Lett. 73, 3495 (1994); Phys. Rev. D 49, 2978 (1994); E.J. Copeland, M. Gleiser and H.-R. Müller, Phys. Rev. D 52, 1920 (1995); M. Gleiser, A. Heckler and E.W. Kolb, Phys. Lett. B 405, 121 (1997); J. Borrill and M. Gleiser, Nucl. Phys. B 483, 416 (1997).

[38] M. Gleiser and A.F. Heckler, Phys. Rev. Lett. 76, 180 (1996).

[39] M. Reuter and C. Wetterich, Nucl. Phys. B 391, 147 (1993); ibid. 408, 91 (1993); ibid. 417, 181 (1994); ibid. 427, 291 (1994).

[40] B. Bergerhoff and C. Wetterich, Nucl. Phys. B 440, 171 (1995); preprints HD-THEP-95-37, hep-ph/9508352 and HD-THEP-96-51, hep-ph/9611462.

[41] W. Buchmüller, Z. Fodor, T. Helbig and D. Walliser, Ann. Phys. 234, 260 (1994); A. Kusenko, Phys. Lett. B 358, 47 (1995).

[42] M. Lindner, Z. Phys. C 31, 295 (1986); M. Sher, Phys. Rep. 179, 273 (1989); M. Lindner, M. Sher and H. Zaglauer, Phys. Lett. B 228, 139 (1989); M. Sher, Phys. Lett. B 317, 159 (1993); G. Altarelli and I. Isidori, Phys. Lett. B 337, 141 (1994); J.A. Casas, J.R. Espinosa and M. Quirós, Phys. Lett. B 342, 171 (1995).

[43] J.R. Espinosa and M. Quirós, Phys. Lett. B 353, 257 (1995). 